\title{
Introduction to the Feature Section on "Crohn's disease activity: MRI assessment and clinical implications"
}

\author{
Francesca Maccioni \\ Department of Radiological Sciences, Policlinico Umberto I, University Sapienza of Rome, Rome, Italy
}

The purpose of this feature section is to provide a clinical-radiological update on an emerging topic of gastrointestinal imaging, the assessment of Crohn's Disease (CD) activity with MRI. The term "Crohn's Disease Activity" is widely used by gastroenterologists to evaluate the degree of intestinal inflammation. Currently, several clinical, biological, endoscopic, and histological indexes are used in the clinical practice to quantify inflammation of CD, including the widely used Crohn's disease activity index (CDAI) and the Crohn's disease endoscopic index of severity (CDEIS). Similarly, several imaging modalities, including contrast-enhanced ultrasonography, contrast-enhanced CT and MRI, are variably used for detecting the main signs of intestinal inflammation in $\mathrm{CD}$. The growing interest shown by gastroenterologists in the assessment of CD activity is related to the need of monitoring the evolution of the disease and the therapeutic efficacy of both traditional and new biological treatments.

Over the last decade, the diagnostic value of MRI in the evaluation of $C D$ activity has been extensively investigated in several clinical-radiological studies. Early radiological papers on "MRI, CD activity" were published in 2000, or shortly before [1-3] as shown in Graph 1 . Since then, the number of scientific papers published on this subject has progressively increased [4-14].

To date, MRI seems to be the most complete and powerful radiological tool to detect inflammatory changes in human tissues and specifically in $\mathrm{CD}$. The high sensitivity of MRI for inflammatory tissues is related to different imaging parameters, mainly fluid-sensitive (T2weighted) and gadolinium-enhanced ones. These MRI parameters allow the detection of intestinal edema and hypervascularity, like no other imaging modality. Furthermore, new imaging parameters and sequences are

Correspondence to: Francesca Maccioni; email: francesca.maccioni@, uniroma1.it continuously added. The last one, diffusion-weighted imaging (DWI), first introduced for the evaluation of neurological diseases, has been recently investigated in the assessment of the activity of inflammatory bowel disease [15-17]. MR molecular imaging and MR spectroscopy are still experimental but extremely promising, due to possibility to analyze and characterize the molecular structure of the inflamed wall. Therefore, MRI has great potentials in characterization of the $\mathrm{CD}$, being able to assess microscopic structural wall changes, including edema and fibrosis, hypervascularity, capillary permeability, diffusion of water molecules, and likely, in the next years, specific molecular abnormalities [15-17].

In detecting signs of intestinal inflammation in $\mathrm{CD}$, MRI competes not only with the other available imaging modalities, but with endoscopy too. The mucosal disease, assessable by endoscopy, represents only the tip of the inflammatory process, whereas the mural and extramural disease, detectable with MRI, is the hidden and submerged part of the inflammation. According to recent studies, MRI has shown the same sensitivity of endoscopy in detecting active intestinal inflammation [5-8, 12].

Since this Feature Section deals with a clinicalradiological subject, a clinical introduction was included, to review the clinical concept of "Crohn's disease activity" [18] and to provide an update on the latest knowledge of the immunological network and the new biologic therapies. Certainly, the concept of CD "activity" needs to be further discussed by radiologists and gastroenterologists. There are too many clinical indexes of activity: purely clinical, biological, endoscopic, and his tological ones [19]. Which of them should be primarily used in the clinical practice? Frequently most of them, if not all, are used. Which one should be considered a reliable gold standard? This is still an open question. When and how the therapeutic planning is influenced by information on CD activity? Furthermore, from a clinical point of view, is there any relevant difference between the "mucosal" 


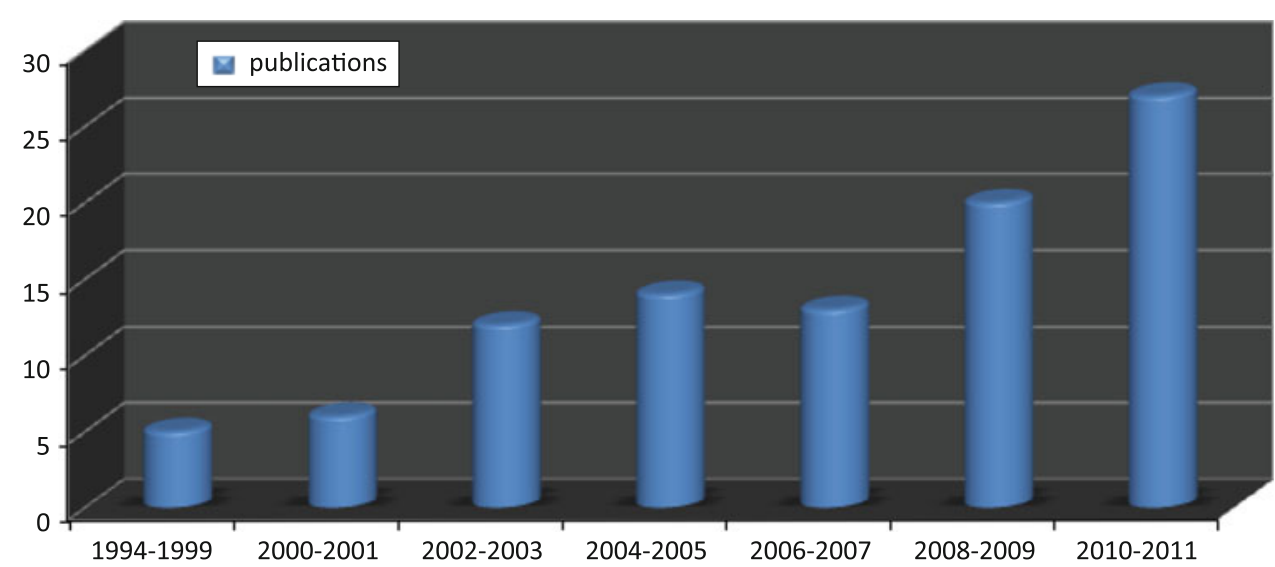

Graph 1. Scientific publications on "MRI, CD activity". A total of approximately 97 scientific papers (both original papers and reviews) have been found in Pub-Med, from 1994 to mid 2011, by using the key words "MRI, Crohn's Disease

healing, assessed by endoscopy, and the "mural" healing, assessed by MRI? Could MRI be considered a reliable gold standard for assessment of CD activity? Could MRI have a prognostic value in addressing the medical treatment?

These and many other open questions will be extensively discussed and they will receive an answer from all clinical and radiological papers contributing to the Feature Section.

In the second and purely radiological part of this session, the actual and future possibilities of MRI in the assessment of CD activity will be reviewed and analyzed.

According to the latest guidelines on imaging of IBD [20], MRI is already considered a reference examination for the assessment of CD activity, together with clinical indexes, ultrasonography, and endoscopy findings. Clinical-radiological guidelines on IBD are currently under revision and new ones are expected in 2012. Undoubtedly, there are still several points to be outlined and defined. At present there is no "radiological agreement" on what should be the best MRI technique and parameters for $\mathrm{CD}$ evaluation. Whether the small bowel should be evaluated with enteroclysis or with oral administration of contrast medium, is still under discussion. Moreover, in the assessment of CD, should MRI evaluate the colon or the small bowel only? Current trends are mainly favorable to the oral administration of the contrast agent with consensual assessment of the small intestine and colon, though the final answer will be likely offered only by the clinical daily practice.

Regardless of this discussion on the MRI technique to be used, the radiological part of the section is mainly focused on the MRI analysis and characterization of the inflammatory intestinal changes of CD. Assessment of disease activity, disease characterization, and detection of wall edema and fibrosis are definitely central issues in the management of $\mathrm{CD}$.
Activity". The columns express the number of papers per couple of years, starting from 2000 to mid 2011. Previous years, between 1994 and 1999, are represented by a single column.

The diagnostic value and usefulness of T1- and T2weighted imaging in the assessment of bowel wall inflammation and in the characterization of Crohn's disease will be specifically analyzed and reviewed in two different papers of the Section [21, 22]. In fact, T1- and T2-weighted parameters can display intestinal wall inflammation, fibrosis and edema in different ways. So far, different morphological and functional parameters, both T1- and T2-weighted, have been tested for the assessment of disease activity including wall thickening, degree of wall Gd-enhancement [3-9, 11, 23], T2 mural signal intensity $[2,3,9,12,13], \mathrm{T} 2$ signal of mesenteric fat [2,9], enhancement of local lymph nodes [24], pattern of wall Gd-enhancement [13], increased mesenteric vascularity [24], and curves of Gd-wall enhancement (washin and wash-out curves) $[8,10]$. Each of these parameters has proved be statistically correlated with the "biological", endoscopic, or histological activity [3-14]. The review of these results should clarify the value of both T1- and T2-weighted imaging in the evaluation of disease activity. The availability of multiple imaging parameters is definitely one of the major advantages of MRI, but it may become a limit, if these parameters are not rationally used with proper imaging techniques. The use of too many parameters in a single MRI examination may be disorienting and confusing. On the other hand, an excessive simplification of the MR techniques could reduce the diagnostic capabilities offered by MRI. A rational and conscious use of the proper imaging parameters is the major issue and task for any radiologist involved in intestinal MRI.

The potential role of each one of these MRI parameters in the development of an MRI index of activity will be also extensively discussed in a specific paper [25]. Recently, a magnetic resonance index of activity (MaRIA) has been proposed [12], highly correlated with the CDEIS. Other indexes have been preliminarily proposed 
or are still under development $[25,26]$. The possibility of an MRI index of activity is extremely appealing, although still to validate with multicenter studies and a consensus conference.

Furthermore, the possibility of a computerized analysis of disease activity, based on well-defined and quantified parameters, is conceivable. The feasibility potentials and drawbacks of a computer-assisted system for the MRI evaluation of disease activity will be extensively discussed [27].

Finally, MRI does not offer only T1- and T2-weighted imaging to assess inflammation. New promising imaging parameters, sequences, and techniques have been recently introduced in the clinical practice or are going to be, including real time MRI, diffusion-weighted parameters, spectroscopy, molecular imaging, and PETMRI [28]. Although the real value of these newest imaging in the evaluation of CD inflammation is still under investigation or has not been tested yet, their potential role will be discussed in the last paper of the Feature Section.

Ideally, the final goal of MRI should be to characterize $\mathrm{CD}$ activity to have a prognostic value in therapeutic planning. A guide in the decision making process is more and more needed, due to the complexity of currently available pharmacological options, frequently very focused and targeted against specific pro-inflammatory cytokines. It is conceivable that in the next years the MRI will offer a key for understanding the effects and efficacy of new biological drugs, providing a guide in the choice of the most effective and early treatments [28].

In conclusion, MRI is certainly a powerful diagnostic tool to assess inflammatory changes in CD. At present, updated MRI systems offer a wide range of imaging parameters to evaluate tissue inflammation. MRI is currently able to analyze and quantify wall vascularity, to differentiate intestinal wall edema and fibrosis, even to detect differences in the motility of water molecules with DWI and metabolic changes by spectroscopy.

Definitely, the potentials of MRI in the detection of CD inflammation are vast, but they can be fully appreciated only at the light of the more recent clinical advances. A strict osmosis between clinical and radiological knowledge will probably be the key of future progresses in clinical-diagnostic research. In the next years, it is expected that radiologists, still maintaining their technical knowledge, will collaborate more and more with gastroenterologists and that, on the other hand, gastroenterologists will be increasingly involved in imaging issues. Likely, the boundaries between clinical and radiological sciences will become less and less defined.

\section{References}

1. Shoenut JP, Semelka RC, Magro CM, et al. (1994) Comparison of magnetic resonance imaging and endoscopy in distinguishing the type and severity of inflammatory bowel disease. J Clin Gastroenterol 19:31-35

2. Madsen SM, Thomsen HS, Schlichting P, et al. (1999) Evaluation of treatment response in active Crohn's disease by low-field magnetic resonance imaging. Abdom Imaging 24:232-239

3. Maccioni F, Viscido A, Broglia L, et al. (2000) Evaluation of Crohn's disease activity with MRI. Abdom Imaging 25:219-228

4. Koh DM, Miao Y, Chinn RJ, et al. (2001) MR imaging evaluation of the activity of Crohn's disease. AJR Am J Roentgenol 177(6):1325-1332

5. Low RN, Sebrechts CP, Politoske DA, et al. (2002) Crohn disease with endoscopic correlation: single shot fast spin echo and gadolinium enhanced fat-suppressed spoiled gradient-echo MR imaging. Radiology 222:652-660

6. Ajaj WM, Lauenstein TC, Pelster G, et al. (2005) Magnetic resonance colonography for the detection of inflammatory diseases of the large bowel: quantifying the inflammatory activity. Gut 54:257263

7. Sempere GAJ, Sanjuan VM, Chulia EM, et al. (2006) MRI evaluation of inflammatory activity in Crohn's disease. AJR Am J Roentgenol 184:1829-18359

8. Florie J, Wasser MN, Arts-Cieslik K, et al. (2006) Dynamic contrast-enhanced MRI of the bowel wall for assessment of disease activity in Crohn's disease. AJR Am J Roentgenol 186(5):1384-1392

9. Maccioni F, Bruni A, Viscido A, et al. (2006) MR imaging in patients with Crohn disease: value of T2- versus T1-weighted gadoliniumenhanced MR sequences with use of an oral superparamagnetic contrast agent. Radiology 238:517-530

10. Del Vescovo R, Sansoni I, Caviglia R, et al. (2008) Dynamic contrast enhanced magnetic resonance imaging of the terminal ileum: differentiation of activity of Crohn's disease. Abdom Imaging 33(4):417-424

11. Horsthuis K, Bipat S, Stokkers PC (2009) Magnetic resonance imaging for evaluation of disease activity in Crohn's disease: a systematic review. Eur Radiol 19:1450-1460

12. Rimola J, Rodriguez S, García-Bosch O, et al. (2009) Magnetic resonance for assessment of disease activity and severity in ileocolonic Crohn's disease. Gut 58(8):1113-1120

13. Punwani S, Rodriguez-Justo M, Bainbridge A, et al. (2009) Mural inflammation in Crohn disease: location-matched histologic validation of MR imaging. Radiology 252:712-720

14. Panés J, Bouzas R, Chaparro M, et al. (2011) Systematic review: the use of ultrasonography, computed tomography and magnetic resonance imaging for the diagnosis, assessment of activity and abdominal complications of Crohn's disease. Aliment Pharmacol Ther 34:125-145

15. Oto A, Kayhan A, Williams JTB, et al. (2011) Active Crohn's disease in the small bowel: evaluation by diffusion weighted imaging and quantitative dynamic contrast enhanced MR imaging. J Magn Reson Imaging 33:615-624

16. Kiryu S, Dodanuki K, Takao H, et al. (2009) Free-breathing diffusion-weighted imaging for the assessment of inflammatory activity in Crohn's disease. J Magn Reson Imaging 29:880-886

17. Oussalah A, Laurent V, Bruo O, et al. (2010) Diffusion-weighted magnetic resonance without bowel preparation for detecting colonic inflammation in inflammatory bowel disease. Gut 59:1056-1065

18. Onali S, Calabrese E, Pallone F, et al. (2012). Measuring disease activity in Crohn's Disease. Abdom Imaging. doi:10.1007/s00261012-9877-3

19. Vucelic B (2009) Inflammatory bowel diseases: controversies in the use of diagnostic procedures. Dig Dis 27(3):269-277

20. Stange EF, Travis SPL, Vermeire S, et al. (2006) European evidence based consensus on the diagnosis and management of Crohn's disease: definitions and diagnosis. Gut 55(Suppl 1):i1-i15

21. Makanyanga J, Punwani S, Taylor SA (2011) Assessment of wall inflammation and fibrosis in Crohn's disease: value of T1-weighted gadolinium-enhanced MR imaging. Abdom Imaging. doi:10.1007/ s00261-011-9821-y

22. Maccioni F, Staltari I, Pino AR, et al. (2012) Value of T2-weighted magnetic resonance imaging in assessment of wall inflammation and fibrosis in Crohn's disease. Abdom Imaging. doi:10.1007/ s00261-012-9853-y

23. Taylor SA, Punwani S, Rodriguez-Justo M, et al. (2009) Mural Crohn disease: correlation of dynamic contrast-enhanced MR 
imaging findings with angiogenesis and inflammation at histologic examination - pilot study. Radiology 251(2):369-379

24. Gourtsoyianni S, Papanikolaou N, Amanakis E (2009) Crohn's disease lymphadenopathy: MR imaging findings. Eur J Radiol 69:425-428

25. Rimola J, Ordás I, Rodríguez S, et al. (2011) Imaging indexes of activity and severity for Crohn's disease: current status and future trends. Abdom Imaging. doi:10.1007/s00261-011-9820-z

26. Maccioni F, Mazzamurro F, Martinelli M et al. (2010) Proposal of an MRI activity index for Crohn's disease, scientific presentation, Abstract ID: 9015491, SS J08-04, RSNA Annual Meeting 2010, Chicago
27. Tielbeek JAW, Vos FM, Stoker J (2011) A computer-assisted model for detection of MRI signs of Crohn's disease activity: future or fiction? Abdom Imaging. doi:10.1007/s00261-011-9822-x

28. Glaudemans AWJ, Maccioni F, Mansi L, et al. (2010) Imaging of cell trafficking in Crohn's Disease. J Cell Physiol 223:562-571 